ITP-SB-94-55

December, 1994

\title{
Models of Fermion Mass Matrices Based on a Flavor- and Generation-Dependent U(1) Gauge Symmetry
}

\author{
Vidyut Jain 母 and Robert Shrock"2 \\ Institute for Theoretical Physics \\ State University of New York \\ Stony Brook, N. Y. 11794-3840
}

\begin{abstract}
We study models of fermion mass matrices based on a flavor- and generation-dependent string-motivated $\mathrm{U}(1)_{A}$ gauge symmetry and report two new classes of solutions to the requisite consistency conditions. In particular, we propose that the fundamental reason underlying the striking feature $m_{b}, m_{\tau}<<m_{t}$ is that all of the elements of the down-quark and charged lepton effective Yukawa matrices actually arise from higher-dimension operators, suppressed by inverse powers of the Plank mass. An explicit model embodying this idea is constructed.
\end{abstract}

\footnotetext{
${ }^{1}$ email: vid@max.physics.sunysb.edu

${ }^{2}$ email: shrock@max.physics.sunysb.edu
} 
The pattern of fermion masses and mixing remains one of the most important mysteries in particle physics. The successful standard model (SM) can accomodate but not explain this pattern. A satisfactory understanding would require that one have an experimentally confirmed theory explaining the related electroweak symmetry breaking (EWSB), and one does not have this at present. Nevertheless, one may proceed by exploring plausible models. The fermion mass spectrum has several striking features: (i) within each charge sector, the masses increase with generation by large factors: $m_{u}<<m_{c}<<m_{t}, m_{d}<<m_{s}<<m_{b}$, and $m_{e}<<m_{\mu}<<m_{\tau}$; (ii) if one assumes that all of these masses arise from conventional, dimension-4 Yukawa couplings, the associated Yukawa couplings for all of these fermions except the top quark are all much smaller than a typical small coupling like $e=\sqrt{4 \pi \alpha} \simeq 0.3$, without explanation; (iii) related to (ii), even if one restricts to the third generation, the masses are still quite different: $m_{\tau}$ and $m_{b}$ are both $<<m_{t}$. A related feature is that (iv) the Cabibbo-Kobayashi-Maskawa (CKM) quark mixing matrix is near to the identity. If one assumes certain simple forms for Yukawa matrices with zeros in various entries, it is possible to explain property (iv) as a consequence of (i), because the quark mixing angles are functions of (square roots of) small mass ratios like $m_{d} / m_{s}, m_{u} / m_{c}$, etc. [1 2]. However, in such an approach, the fermion masses are used as inputs, and properties (i), (ii), and (iii) are not explained.f Indeed, although there has been recent progress in explaining (i) and (iv) via contributions of higher-dimension operators at a high mass scale near to the scale of quantum gravity [3], such efforts have not addressed what, to us, seems an equally remarkable feature, viz., (iii). The attractive idea of radiative electroweak symmetry breaking in a supersymmetric generalization of the standard model [⿴囗十 depends on the existence of at least one quark which has a mass comparable to the EWSB scale, but it cannot explain why this was the top quark instead of the bottom quark (or, indeed why both $m_{t}$ and $m_{b}$ are not $\sim$ the EWSB scale), and hence it cannot explain (iii) or the full extent of (ii).

In this paper, we shall explore an appealing class of models of fermion mass matrices which has the potential to explain all of the properties (i)-(iv). We shall present a particular model which, we believe, is the first to offer a possible fundamental explanation of property (iii). The explanation is that in the down-quark and charged lepton sectors, the masses of not just the first two generations, but of all generations arise from higher-dimension operators suppressed by powers of a small mass ratio, $\epsilon \propto v / \bar{M}_{P}$, where $v$ is the breaking scale of a flavor- and generation-dependent, string-motivated $\mathrm{U}(1)_{A}$ symmetry, and $\bar{M}_{P} \equiv$ $\left(8 \pi G_{N}\right)^{-1 / 2}=2.44 \times 10^{18} \mathrm{GeV}$ is the (reduced) Planck mass.

To set our work in context, we note that since the original success of the standard model, there has been a growing appreciation that its renormalizability and, in particular, the absence of higher-dimension operators, may well be the consequence of a large logarithmic interval in energy between the electroweak scale and a higher scale where there is new physics (see, e.g., Ref. [5]). Indeed, there are specific reasons for expecting such operators at this high scale: the only known way to stabilize the hierarchy $v_{E W}<<\bar{M}_{P}$ (where $v_{E W}=246 \mathrm{GeV}$ is the EWSB scale) is via a supersymmetric generalization of the SM. In turn, global supersymmetry is naturally embedded in supergravity, which one also finds as

\footnotetext{
${ }^{1}$ Indeed, the model of Ref. [2] is excluded by property (iii), since in that model, $\left|V_{c b}\right| \simeq \mid \sqrt{m_{s} / m_{b}}-$ $e^{i \phi} \sqrt{m_{c} / m_{t}} \mid$ (masses normalized at same scale $\mu$ ); because $m_{t}>>m_{c}$, the second term is too small to significantly cancel the first term $\sqrt{m_{s} / m_{b}} \simeq 0.17$ and fit the experimental value, $\left|V_{c b}\right|=0.038 \pm 0.005$.
} 
the low-energy limit of the main candidate for quantum gravity, string theory. But $d=4$ supergravity is nonrenormalizable. Indeed, explicit calculations of the pointlike limit of string theories for energies $E<<M_{s t r}$ (where $M_{s t r}=2\left(\alpha^{\prime}\right)^{-1 / 2}=g \bar{M}_{P}$ ) yield supergravity as a lowenergy effective field theory with infinite towers of higher-dimension operators with coefficient functions proportional to inverse powers of $M_{s t r}$ and powers of the compactification scale (the latter may be non-explicit in four-dimension string formulations). One must therefore take account of higher-dimension operators when analyzing terms which contribute to fermion mass matrices. This was, indeed, already realized long ago [6], although detailed studies have only been carried out recently. At first, one might consider these higher-dimension operators to be an unfortunate, if inevitable, complication in the theory. However, they may well play a very important role in the area of fermion masses. Specifically, via vacuum expectation values (vev's) of the scalar components of certain chiral superfields, which we shall denote generically as $v$, these higher-dimension operators can yield contributions to effective dimension-4 Yukawa interactions which are suppressed by powers of the ratio $\epsilon \sim$ $v / M_{s t r}$. This idea has already been used for a possible explanation of properties (i) and (iv) [3]; here we shall extend this work with new solutions of the consistency conditions and take a step further, to use the small ratio $\epsilon$ to explain (ii) and (iii). A preliminary report of some of our results was given in Ref. [7]. Related results in a somewhat different direction (having $m_{b}$ and $m_{\tau}$ arise from dimension-4 operators, as in Ref. [3] ) were presented in Ref. [8].

We shall work within the context of a supergravity theory which reduces at low energies to the minimal supersymmetric standard model (MSSM). We consider a theory where there is a flavor and generational symmetry group $G_{F}$ which restricts the forms of the terms in the action. In particular, this symmetry forbids certain cubic superfield couplings which give rise to Yukawa interactions. Since this happens at a scale not too far from that of quantum gravity, and since, in general, global symmetries are broken by quantum gravity [9] even at the semi-classical level, one is motivated to make $G_{F}$ a gauge symmetry. One then faces two questions: (a) is there a natural origin for $G_{F}$ in the presumed underlying string theory? and (b) is there a natural way to explain why the scale of the breaking of $G_{F}$ is such that the ratio $v / M_{s t r}$ has the value that it must to fit the observed forms of fermion mass matrices? A possible affirmative answer to both of these questions is provided by a gauged symmetry $G_{F}=U(1)_{A}$ which is, at the field theory level, apparently anomalous, but whose anomaly is cancelled by a Green-Schwarz mechanism [10]. Such $\mathrm{U}(1)_{A}$ gauge symmetries are known to arise is various string models and, moreover, they are broken at a calculable scale $v$ given by $v^{2} \simeq M_{\text {str }}^{2} /\left(192 \pi^{2}\right)$ [11], so that $\epsilon \sim(8 \pi \sqrt{3})^{-1}=0.023$, a value which is in the right general range to explain fermion mass hierarchies [3]. Of course, such a $\mathrm{U}(1)_{A}$ symmetry does not mix up-type and down-type quark superfields, or mix these with lepton superfields and is thus quite different from flavor and generational symmetries which comprise extensions of grand unified groups.

We shall denote the (left-handed) SM matter chiral superfields as $Q_{i}, u_{i}^{c}, d_{i}^{c}, L_{i}$, and $e_{i}^{c}$, where $i=1,2,3$ is the generation, with $\left\{u_{i}\right\}=\{u, c, t\},\left\{d_{i}\right\}=\{d, s, b\}$, etc. Under the flavor- and generation-dependent $\mathrm{U}(1)_{A}$, these carry the charges $q_{Q_{i}}, q_{u_{i}^{c}}, q_{d_{i}^{c}}, q_{L_{i}}$, and $q_{e_{i}^{c}}$. The $Y=1,-1$ Higgs chiral superfields are denoted $H_{1}$ and $H_{2}$ and have $\mathrm{U}(1)_{A}$ charges $q_{H_{1}}$ and $q_{H_{2}}$. We shall also assume that the theory is invariant under the usual $R$ parity. The 
cubic superfield terms in the globally supersymmetric superpotential are then given by

$$
W_{\text {cubic }}=Q_{i} Y_{i j}^{(u)} u_{j}^{c} H_{2}+Q_{i} Y_{i j}^{(d)} d_{j}^{c} H_{1}+L_{i} Y_{i j}^{(e)} e_{j}^{c} H_{1}
$$

We shall assume that the gauge symmetry at energies $\gtrsim v$ is $G=G_{S M} \times \mathrm{U}(1)_{A}$, where $G_{S M}$ is the $\mathrm{SM}$ gauge group $\mathrm{SU}(3) \times \mathrm{SU}(2) \times \mathrm{U}(1)_{Y}$. The theory will also contain certain chiral superfields which are SM-singlets but transform under the $\mathrm{U}(1)_{A}$. A discussion of constraints on these to avoid destabilization of the gauge hierarchy is given in Ref. [12].

To proceed, we recall how the cancellation of an apparent field-theoretic anomaly in a gauged $\mathrm{U}(1)$ symmetry works when this $\mathrm{U}(1)$ is, in fact, non-anomalous in the full string theory (Green-Schwarz mechanism) [10 and the related (Dine-Seiberg-Witten, DSW) mechanism whereby the $\mathrm{U}(1)$ is broken [1]. Here, we shall denote chiral superfields generically by $\Phi$. Standard $d=4$ supergravity is described by two functions. The first is the generalized Kähler potential

$$
G(\Phi, \bar{\Phi})=K(\Phi, \Phi)+\ln W(\Phi)+\ln \bar{W}(\bar{\Phi})
$$

is a hermitian function of the Kähler potential $K$ and the superpotential $W$, the latter being a holomorphic function of the chiral superfields. The second is the gauge kinetic normalization (matrix) function $f_{a b}(\Phi)$, where $a, b=1, . ., N_{G}$, the number of gauge bosons. As indicated, $f_{a b}$ is a holomorphic function of the chiral superfields. We shall use $G, K$, and $W$ to denote both superfield quantities and also their scalar components; however we shall distinguish between chiral superfields and their scalar components by type case (e.g. $\phi=\Phi|, m=M|$ ).

The bosonic part of the standard supergravity lagrangian is [14]

$$
\frac{1}{\sqrt{g}} \mathcal{L}_{B}=G_{i \bar{j}} D_{\mu} \phi^{i} g^{\mu \nu} D_{\nu} \phi^{\bar{j}}+\frac{1}{2} R-\frac{1}{4} \operatorname{Re} f_{a b} F_{\mu \nu}^{a} F^{b \mu \nu}-\frac{1}{4} \operatorname{Im} f_{a b} F_{\mu \nu}^{a} \tilde{F}^{b \mu \nu}-V,
$$

where the auxiliary field contributions to the potential are given by $V=\hat{V}+\mathcal{D}$, with

$$
\hat{V}=e^{G}\left(G_{i} G_{\bar{j}} G^{i \bar{j}}-3\right)
$$

and

$$
\mathcal{D}=\operatorname{Re}\left(f_{a b}^{-1}\right) D^{a} D^{b},
$$

where $D_{a}$ is the D-type auxiliary field associated with generators $T_{a}$ of the gauge group, normalized according to $\operatorname{Tr}\left[T_{a}, T_{b}\right]=(1 / 2) \delta_{a b}$. Discussions of one-loop corrections are in Ref. [15 and references therein. The scalar fields and their conjugates are denoted as above by $\phi^{i}$ and $\phi^{\bar{i}}=\bar{\phi}^{i}$. The Kähler metric $G_{i \bar{j}}=K_{i \bar{j}}=\partial_{\phi^{i}} \partial_{\phi^{j}}$ has inverse $G^{i \bar{j}}$. Finally, the derivatives $D_{\mu}$, which are gauge and general coordinate invariant, are normalized so that $D_{\mu}=\partial_{\mu}+\sum_{a} i A_{\mu}^{a} T_{a}$.

The function $f_{a b}$ determines the gauge coupling constants of the theory and also plays an important role in the cancellation of the field theoretic anomaly due to the $F \tilde{F}$ term in the Lagrangian. In string models, at tree level, $f_{a b}$ is given by

$$
f_{a b}=k_{a} s \delta_{a b}
$$

where the levels $k_{a}$ of the Kac-Moody algebras on the worldsheet depend on the gauge factor group $G_{a}$ [16. We denote $k_{i}, i=1,2,3$ and $k_{A}$ respectively as the Kac-Moody levels 
corresponding to the factor groups $\mathrm{U}(1)_{Y}, \mathrm{SU}(2)$, and $\mathrm{SU}(3)$ of $G_{S M}$ and the flavor symmetry $\mathrm{U}(1)_{A}$. The dilaton vev determines the gauge couplings, according to $1 / g_{a}^{2}=k_{a}<\operatorname{Re}(s)>$. At one- and higher-loop levels, $f_{a b}$ acquires a dependence also on the moduli fields [17, 18, 19] from chirally anomalous triangle diagrams in the effective field theory as well as string threshold corrections generically needed to cancel these anomalous terms, as is required by the underlying string theory. However, there is a large class of models in which the field theoretic chiral anomalies can be cancelled by a "universal" Green-Schwarz mechanism involving the dilaton, similar to the gauge $U(1)$ anomaly cancellation, in which the one-loop corrected $f_{a b}$ is such that $\operatorname{Re}\left(f_{a b}\right)$ (which is what controls the unification conditions for the gauge couplings) has the form $\operatorname{Re}\left(f_{a b}\right)=k_{a} \delta_{a b}(\operatorname{Re}(s)+f(T, \bar{T}))$, where $T$ denotes the moduli [17, 18, 19. We shall restrict our attention to this class of models. (There are also small logarithmic corrections from the running of the gauge couplings between $M_{s t r}$ and $M_{X}$, the scale of gauge coupling unification; as in [3], we shall neglect these here since $M_{X}$ is not $<<M_{\text {str. }}$ )

If the effective theory contains no gauge anomalies, then (3) is gauge-invariant, with the dilaton being a gauge singlet. In the case of interest here, however, where $G$ contains a $U(1)_{A}$ factor which has field theoretic anomalies, then one-loop corrections from light $\left(<\bar{M}_{P l}\right)$ fermions give an anomalous correction $\mathcal{L}_{\text {anom }}$ to (3). Under a $\mathrm{U}(1)_{A}$ transformation, $\mathcal{L}_{\text {anom }}$ transforms by

$$
\delta \mathcal{L}_{\text {anom }} \propto c_{A} F_{A}^{\mu \nu} \tilde{F}_{\mu \nu}^{A}+\sum_{a} c_{a} F_{a}^{\mu \nu} \tilde{F}_{\mu \nu}^{a}+\ldots
$$

where

$$
\begin{gathered}
c_{A}=(1 / 3) \operatorname{Tr}\left(T_{A} T_{A} T_{A}\right) \\
c_{a}=\operatorname{Tr}\left(T_{A} T_{a} T_{a}\right),
\end{gathered}
$$

and $a$ runs over the gauge group factors which have no field theoretic anomalies (in the present case, the factors in $\left.G_{S M}\right)$. The ellipses in (5) indicate additional terms which we shall discuss below.

The theory is invariant under constant rescalings $A_{a}^{\mu} \rightarrow \alpha A_{a}^{\mu}$, which amounts to a simultaneous redefinition of $k_{a}$ and $T_{a}: k_{a} \rightarrow \alpha^{2} k_{a}$ and $T_{a} \rightarrow \alpha T_{a}$. (Our normalization of the generators of $G_{S M}$ is the standard one.) For the $U(1)_{A}$ group the above rescaling could be used to set $k_{A}$ to 1 at the expense of modifying the $U(1)_{A}$ charges. We shall instead use this rescaling to fix the $U(1)_{A}$ charge of one of the SM-singlet particles.

Due to the coupling of $\operatorname{Im}(s)$ to $F \tilde{F}$ in (3), this variation of $\mathcal{L}_{\text {anom }}$ can be cancelled by assigning a $U(1)_{A}$ gauge variation to $\operatorname{Im}(s)$ [10, 11, but only if

$$
c_{A}: c_{a}:: k_{A}: k_{a} \text { for all } a \text {. }
$$

Since $s$ is no longer a gauge singlet, its kinetic terms must be made gauge invariant. At the superfield level, this means that the Kähler function $K$ must include a coupling between the chiral superfield $S$ and $V_{A}$, the real superfield that contains the $U(1)_{A}$ gauge multiplet. Specifically, the tree level Kähler function for $S$ is modified as [11]

$$
\begin{aligned}
-\ln (S+\bar{S}) & \rightarrow-\ln \left(S+\bar{S}+c V_{A}\right) \\
& =-\ln (S+\bar{S})-\frac{c V_{A}}{S+\bar{S}}+\frac{c^{2} V_{A}^{2}}{2(S+\bar{S})^{2}}+\ldots
\end{aligned}
$$


where $c$ is a constant determined by gauge invariance and is related to the coefficients (9). The second term in the last line of (11) produces a term in $\mathcal{L}_{B}$ which is linear in the auxiliary $D$ field of $V_{A}$. Once $\operatorname{Re}(s)$ acquires a nonzero vev, this causes some of the fields which are charged under the $\mathrm{U}(1)_{A}$ to acquire vev's, thereby spontaneously breaking $\mathrm{U}(1)_{A}$.

In a given string model, (10) is automatically satisfied, due to the absence of gauge anomalies in the underlying theory. The absence of anomalous terms that mix the different gauge group factors requires that

$$
\operatorname{Tr}\left(T_{A} T_{A} T_{a}\right)=0 \text { for all } a
$$

This ensures that $\delta \mathcal{L}_{\text {anom }}$ does not contain any $F \tilde{F}$ terms that cannot be cancelled by a gauge variation of $s$. Furthermore, mixed gauge and gravitational anomalies must be absent. In addition to terms explicitly displayed in (11), the $\mathrm{U}(1)_{A}$ gauge variation of $\mathcal{L}_{\text {anom }}$ contains a term proportional to $\operatorname{Tr}\left(T_{A}\right) R^{\mu \nu} \tilde{R}_{\mu \nu}$. This term can be cancelled because the effective Lagrangian contains a nonstandard (higher derivative) coupling $k_{G} \operatorname{Im}(s) R^{\mu \nu} \tilde{R}_{\mu \nu}$ (which defines $\left.k_{G}\right)$. The cancellation requires a relation between the coefficients $c_{a}, c_{A}$ and $\operatorname{Tr}\left(T_{A}\right)$ because the same $U(1)_{A}$ transformation of $s$ must cancel all anomalies. This last relation is difficult to use phenomenologically because (i) $\operatorname{Tr}\left(T_{A}\right)$ depends on SM singlets about which we have very little experimental information, and (ii) because it depends on $k_{A}$ and $k_{G}$ which are not measured at low energies.

We proceed to investigate such models to obtain phenomenologically acceptable fermion mass matrices. Within the above theoretical framework, we shall make the following specific assumptions:

1. The low energy theory near the electroweak scale is the MSSM with phenomenologically viable soft supersymmetry breaking and supersymmetric mass terms. This assumption precludes SM nonsinglets which carry $U(1)_{A}$ charge from getting large vev's due to the $U(1)_{A} D$-term.

2. Supersymmetry is spontaneously broken in a hidden sector with $m_{3 / 2}^{2}=<e^{G}>\sim m_{W}^{2}$ and $<V>\sim 0$; i.e., $O\left(M_{P}^{4}\right)$ and $O\left(M_{P}^{2} m_{3 / 2}^{2}\right)$ contributions to $\langle V\rangle$ cancel. This ensures that the soft breaking terms are naturally of $O\left(m_{W}\right)$. In addition, this assumption means that the dominant contributions to the effective Yukawa couplings are only from superpotential terms, as in the MSSM, and not from $K$, which can also potentially contribute to the effective Yukawa couplings in a supergravity model [20].

3. The SM gauge couplings unify in the canonical way, i.e., $g_{3}^{-2}: g_{2}^{-2}: g_{Y}^{-2}=1: 1: 5 / 3$, or equivalently, $k_{3}: k_{2}: k_{1}=1: 1: 5 / 3$.

4. SM singlet fields $\chi$ which get a vev with $\left\langle D_{A}\right\rangle=0$ carry $U(1)_{A}$ charges with the same sign. In the class of models that we consider, $\left\langle\chi>\sim 10^{16} \mathrm{GeV}\right.$, which is $>>m_{W}$. This ensures that $\langle W\rangle$ (and hence $\left\langle e^{G}\right\rangle$ ) can be kept small without resorting to accidental near-cancellation between two large vev's in $\langle W\rangle$ or introducing extra symmetry. For example, if the $U(1)_{A}$ charge of $\chi$ is 1 and the $U(1)_{A}$ charge of another SM singlet $\chi^{\prime}$ is $-b, b=2,3, \ldots$, then gauge invariance allows $W$ to contain terms of 


\begin{tabular}{|c|c|c|c|c|c|}
\hline \hline$f$ & $Q$ & $u^{c}$ & $d^{c}$ & $L$ & $e^{c}$ \\
\hline \hline$q_{f_{1}}$ & $\bar{q}_{Q}+\alpha_{1}$ & $\bar{q}_{u^{c}}+\beta_{1}$ & $\bar{q}_{d^{c}}+\gamma_{1}$ & $\bar{q}_{L}+a_{1}$ & $\bar{q}_{e^{c}}+b_{1}$ \\
$q_{f_{2}}$ & $\bar{q}_{Q}+\alpha_{2}$ & $\bar{q}_{u^{c}}+\beta_{2}$ & $\bar{q}_{d^{c}}+\gamma_{2}$ & $\bar{q}_{L}+a_{2}$ & $\bar{q}_{e^{c}}+b_{2}$ \\
$q_{f_{3}}$ & $\bar{q}_{Q}-\left(\alpha_{1}+\alpha_{2}\right)$ & $\bar{q}_{u^{c}}-\left(\beta_{1}+\beta_{2}\right)$ & $\bar{q}_{d^{c}}-\left(\gamma_{1}+\gamma_{2}\right)$ & $\bar{q}_{L}-\left(a_{1}+a_{2}\right)$ & $\bar{q}_{e^{c}}-\left(b_{1}+b_{2}\right)$ \\
\hline
\end{tabular}

Table 1: $\mathrm{U}(1)_{A}$ charges of SM matter chiral superfields $f_{i}$.

the form $\chi^{b} \chi^{\prime} / \bar{M}_{P}^{(b-2)}$. If both $\chi$ and $\chi^{\prime}$ get vev's $\sim \bar{M}_{P} / 100$ then the contribution of this term to $\langle W\rangle$ is phenomenologically too large, unless $b$ is sufficiently large.

5. To reduce the number of parameters, it is convenient to assume that the effective Yukawa matrices are symmetric at $M_{X}$.

The hierarchical structure of the low-energy fermion masses can be produced by Yukawa couplings which are either hierarchical or democratic. In the latter case, all entries of a Yukawa matrix are the same to leading order, while subleading corrections to these differ so that the eigenvalues of the matrix have the desired hierarchical structure. A $U(1)_{A}$ symmetry can potentially explain hierarchical Yukawa matrices but not (by itself) democratic matrices. In contrast, a hierarchical structure can be produced by assigning $U(1)_{A}$ charges to the operators in (1) in a manner such that for different $i, j$, these couple to different requisite powers of certain SM singlet chiral superfields.

We next determine which $\mathrm{U}(1)_{A}$ charge assignments for the fields satisfy the various constraints and lead to experimentally viable fermion Yukawa matrices. There are $N_{q}=$ $5 N_{G}+2=17 \mathrm{U}(1)_{A}$ charges in the MSSM, where $N_{G}=3$ is the number of matter generations. In addition, there are $N_{s} \mathrm{U}(1)_{A}$ charges for SM-singlet chiral superfields. The conditions (10) and (12), together with the assumption of symmetric mass matrices, reduce these 17 parameters to 8 . To show this, we first list the $\mathrm{U}(1)_{A}$ charges of the SM matter fields as in Table 1, where $\bar{q}_{f}=(1 / 3) \sum_{i=1}^{3} q_{f_{i}}$ is the generational average charge for the $f$ chiral superfield. For the Higgs chiral superfields, we put $q\left(H_{i}\right)=q_{H_{i}}, i=1,2$. To ensure symmetric Yukawa matrices, we require that the $\mathrm{U}(1)_{A}$ charges of the chiral superfield bilinears satisfy

$$
\begin{aligned}
q\left(Q_{i} u_{j}^{c}\right) & =q\left(Q_{j} u_{i}^{c}\right) \\
q\left(Q_{i} d_{j}^{c}\right) & =q\left(Q_{j} d_{i}^{c}\right) \\
q\left(L_{i} e_{j}^{c}\right) & =q\left(L_{j} e_{i}^{c}\right)
\end{aligned}
$$

In all, there are six independent contraints in these equations. The solutions are

$$
\alpha_{i}=\beta_{i}=\gamma_{i}, \quad a_{i}=b_{i}, \text { for } i=1,2
$$

Because the anomalies $c_{i}$ in eq. (9) are linear in the $\mathrm{U}(1)_{A}$ charges, it follows that, regarding the contributions from the matter fields, the $c_{i}$ only depend on the generational average $\mathrm{U}(1)_{A}$ charges. These anomalies are

$$
U(1)_{Y}^{2} U(1)_{A}:(1 / 4)\left[N_{G}\left\{(2 / 3) \bar{q}_{Q}+(16 / 3) \bar{q}_{u^{c}}+(4 / 3) \bar{q}_{d^{c}}+2 \bar{q}_{L}+4 \bar{q}_{e^{c}}\right\}+2 q_{H_{1}}+2 q_{H_{2}}\right]
$$




$$
\begin{gathered}
S U(2)^{2} U(1)_{A}: c_{2}=(1 / 2)\left[N_{G}\left(3 \bar{q}_{Q}+\bar{q}_{L}\right)+q_{H_{1}}+q_{H_{2}}\right] \\
S U(3)^{2} U(1)_{A}: c_{3}=\left(N_{G} / 2\right)\left(2 \bar{q}_{Q}+\bar{q}_{u^{c}}+\bar{q}_{d^{c}}\right)
\end{gathered}
$$

where we show the general $N_{G}$ dependence but take $N_{G}=3$ here. The anomaly conditions (10) yield two linearly independent constraints on the 7 parameters $\bar{q}_{Q}, \bar{q}_{u^{c}}, \bar{q}_{d^{c}}, \bar{q}_{L}, \bar{q}_{e^{c}}, q_{H_{1}}$, and $q_{H_{2}}$, e.g., $c_{2}=c_{3}$ and $c_{1}=(5 / 3) c_{2}$. These can be solved in terms of the 5 quantities $x$, $y, z, v$, and $w$ according to

$\bar{q}_{Q}=v+x, \quad \bar{q}_{u^{c}}=2 v+x, \quad \bar{q}_{d^{c}}=w+y, \quad \bar{q}_{L}=y, \quad \bar{q}_{e^{c}}=x, \quad q_{H_{1}}=3(v+w)+z, \quad q_{H_{2}}=-z$

The $\mathrm{U}(1)_{Y} \mathrm{U}(1)_{A}^{2}$ anomaly (21) is quadratic in the $\mathrm{U}(1)_{A}$ charges, and hence, in general, cannot be written just as a function of the generational averages of the matter field charges. However, given (13)-(15), this anomaly also depends only on these averages; requiring that it vanish gives

$$
U(1)_{Y} U(1)_{A}^{2}: 0=c_{Y A A}=N_{G}\left(\bar{q}_{Q}^{2}-2 \bar{q}_{u^{c}}^{2}+\bar{q}_{d^{c}}^{2}-\bar{q}_{L}^{2}+\bar{q}_{e^{c}}^{2}\right)+q_{H_{2}}^{2}-q_{H_{1}}^{2}
$$

In terms of the 5 quantities in eq. (20), eq. (21) is

$$
0=2\left(w^{2}-v^{2}\right)+3 v(w-x)+v z+w(y+z)
$$

We find the following three families of solutions to (22), which are thus solutions to the total set of anomaly constraints (these solutions are independent of $N_{s}$ ):

$$
\begin{array}{ccccccc}
\bar{q}_{Q} & \bar{q}_{u^{c}} & \bar{q}_{d^{c}} & \bar{q}_{L} & \bar{q}_{e^{c}} & q_{H_{2}} & q_{H_{1}} \\
x & x & y & y & x & z & -z \\
x & x & \frac{1}{2}(y-z) & y & x & -\frac{1}{2}(3 y+z) & -z \\
x+v & x+2 v & y+w & y & x & 3(v+w)+z & -z
\end{array}
$$

The first two correspond to $v=0$ and describe two distinct 3-parameter families of solutions. The last exists for $v \neq 0$ and describes a 4-parameter family of solutions with $x$ solved for in (22). The first solution in (23) was already given in Ref. [3]; the other two were not mentioned there and are a new result in the present work. The four parameters $\alpha_{1}, \alpha_{2}, a_{1}, a_{2}$, together with the unknown parameters in (23) yield all allowed $\mathrm{U}(1)_{A} \mathrm{SM}$ charges consistent with our assumptions. We find that the constraints are very restrictive, as will be seen.

In order to account for the important feature (ii) that $m_{t}$ is comparable to the EWSB scale $v_{E W}$, one chooses the source of $m_{t}$ to be a renormalizable, dimension- 4 Yukawa coupling, as in the SM. This requires the $\mathrm{U}(1)_{A}$ charge of $Q_{3} u_{3}^{c} H_{2}$ to be zero, i.e.,

$$
\bar{q}_{Q}+\bar{q}_{u^{c}}+q_{H_{2}}=2\left(\alpha_{1}+\alpha_{2}\right)
$$

Let us denote the $\mathrm{U}(1)_{A}$ charge of $\left(Q_{3} d_{3}^{c} H_{1}\right)$ as $\theta$. Then

$$
\bar{q}_{Q}+\bar{q}_{d^{c}}+q_{H_{1}}=2\left(\alpha_{1}+\alpha_{2}\right)+\theta .
$$

Under this assumption, the $U(1)_{A}$ charges of $\left(Q_{i} u_{j}^{c} H_{2}\right)$ are given by the matrix

$$
\left(\begin{array}{ccc}
4 \alpha_{1}+2 \alpha_{2} & 3 \alpha_{1}+3 \alpha_{2} & 2 \alpha_{1}+\alpha_{2} \\
3 \alpha_{1}+3 \alpha_{2} & 4 \alpha_{2}+2 \alpha_{1} & 2 \alpha_{2}+\alpha_{1} \\
2 \alpha_{1}+\alpha_{2} & 2 \alpha_{2}+\alpha_{1} & 0
\end{array}\right)
$$


while

$$
q\left(Q_{i} d_{j}^{c} H_{1}\right)=q\left(Q_{i} u_{j}^{c} H_{2}\right)+\theta .
$$

If one took $\theta=0$, then the $b$ quark mass would arise from a renormalizable cubic superfield operator, and one would not have any fundamental explanation of property (iii). The origin of the large mass ratio $m_{t} / m_{b}$, rather than being explained naturally, would have to be pushed into a similarly large value of $\tan \beta=v_{2} / v_{1}$, viz., $\tan \beta \sim m_{t} / m_{b}$. Instead, we take $\theta \neq 0$, implementing our explanation of properties (ii) and (iii), since then $m_{b}$ arises from higher-dimension operators, and $m_{b}<<m_{t}$ follows naturally.

We proceed to construct an explicit model for fermion mass matrices embodying these ideas. We assume that there are $N_{s}=2$ chiral superfields which are SM singlets (and $U(1)_{A}$ nonsinglets), $\chi$ and $\chi^{\prime}$ with unequal $U(1)_{A}$ charges $q_{\chi}$ and $q_{\chi^{\prime}}$. For convenience, let us normalize $q_{\chi}=1$ (this can be done by rescaling the $\mathrm{U}(1)_{A}$ coupling) and define $q_{\chi^{\prime}} \equiv \theta^{\prime}$. The DSW breaking of the $\mathrm{U}(1)_{A}$ yields values for $\left\langle\chi>/ \bar{M}_{P} \sim<\chi^{\prime}>/ \bar{M}_{P}\right.$ (denoted $\epsilon$ above) which are $\sim O\left(\lambda^{2}\right)$, where $\lambda=\left|V_{u s}\right| \simeq 0.22$ is a measure of the hierarchical structure of the CKM matrix. First, consider the up- and down-quark masses. We have

$$
W \ni \mathcal{Y}_{i j}^{u}\left(\chi, \chi^{\prime}\right) H_{2} Q_{i} u_{j}^{c}+\mathcal{Y}_{i j}^{d}\left(\chi, \chi^{\prime}\right) H_{1} Q_{i} d_{j}^{c},
$$

so that $Y_{i j}^{u}=<\mathcal{Y}_{i j}^{u}>$ and $Y_{i j}^{d}=<\mathcal{Y}_{i j}^{d}>$. Motivated by string theory considerations, we shall consider only functions $\mathcal{Y}$ which do not contain fractional or negative powers of $\chi$ and $\chi^{\prime}$. In order to obtain a viable form of the quark mass matrices, we require $Y_{22}^{u} \sim \lambda^{4}$, i.e. $\left(\mathcal{Y}_{22}^{u} \sim \chi^{2}, \chi^{\prime 2}\right.$ or $\left.\chi^{\prime} \chi\right)$ or $\left(Y_{23}^{u} \sim \lambda^{2}\right.$, i.e. $\mathcal{Y}_{23}^{u} \sim \chi$ or $\left.\chi^{\prime}\right)$.

We consider three cases:

(1) $\mathcal{Y}_{22}^{u} \sim \chi \chi^{\prime} . \mathrm{U}(1)_{A}$ charge conservation $(\mathrm{CC})$ then implies $\mathcal{Y}_{23}^{u}=\mathcal{Y}_{32}^{u} \neq \chi, \chi^{\prime}$.

(2) $\mathcal{Y}_{23}^{u} \sim \chi$. Then $\mathrm{CC} \Rightarrow \mathcal{Y}_{22}^{u} \sim \chi^{2}$.

(3) $\mathcal{Y}_{23}^{u} \sim \chi^{\prime}$. Then $\mathrm{CC} \Rightarrow \mathcal{Y}_{22}^{u} \sim \chi^{\prime 2}$.

We have studied all of these cases and find in case (2) an assignment of $\mathrm{U}(1)_{A}$ charges which yields effective Yukawa matrices (i.e., the matrices which enter in effective dimension-4 Yukawa terms, all of which, except for $Y_{33}^{u}$, actually arise from higher-dimension operators) which give an acceptable pattern of fermion masses at the electroweak scale. At $M_{X}$, these are close to the simple forms

$$
Y^{(u)} \propto\left(\begin{array}{ccc}
0 & \lambda^{6} & 0 \\
\lambda^{6} & 0 & \lambda^{2} \\
0 & \lambda^{2} & 1
\end{array}\right), \quad Y^{(d)} \propto\left(\begin{array}{ccc}
0 & \lambda^{4} & 0 \\
\lambda^{4} & \lambda^{3} & \lambda^{3} \\
0 & \lambda^{3} & 1
\end{array}\right),
$$

where the actual entries in the positions given by zeros in (29) need not be, and are not in general, exactly zero; indeed, one may have $Y_{22}^{(u)} \sim \lambda^{4}, Y_{11}^{(u)} \lesssim O\left(\lambda^{8}\right), Y_{13}^{(u)} \lesssim O\left(\lambda^{4}\right)$, and so on (e.g. [3]). The solution we give below satisfies these bounds. In writing such forms, it is understood that the coefficients $a_{i j}$ multiplying a given power of $\lambda$ may differ from unity, but not by as much as a positive or negative integer power of $\lambda$. The pattern (29) is known to be experimentally viable [21, 22], and our $\mathrm{U}(1)_{A}$ charge assignments constitute a new way of obtaining this pattern.

Consider two cases

(2a) $\theta=-1$. Then, as for case (1a), $\mathcal{Y}_{i j}^{d}=\chi \mathcal{Y}_{i j}^{u}+\tilde{\mathcal{Y}}_{i j}^{d}$. Since $Y_{22}^{u} \sim \lambda^{4}, Y_{23}^{u}=Y_{32}^{u} \sim \lambda^{2}$, 
$Y_{33}^{u} \sim 1$ and $Y_{23}^{d}=Y_{32}^{d} \sim \lambda^{4}+<\tilde{\mathcal{Y}}_{23}^{d}>$, we must fit (29). We have $Y_{22}^{d} \sim \lambda^{6}+<\tilde{\mathcal{Y}}_{22}^{d}>$ and we need $Y_{22}^{d} \sim \lambda^{4}$, so we require $\tilde{\mathcal{Y}}_{22}^{d} \sim \chi^{b} \chi^{\prime(2-b)}$ for $b-0,1$, 2 . Since $4 \alpha_{2}+2 \alpha_{1}=-2$, CC $\Rightarrow$

$$
b+\theta^{\prime}(2-b)-3=0 .
$$

This cannot be satisfied for $b=2$, while for $b=1, \theta^{\prime}=2$ which would mean $\mathcal{Y}_{22}^{u} \sim \chi^{\prime}, \chi^{2}$ or $Y_{22}^{u} \sim \lambda^{2}+\ldots$ which is too big. Hence, the only acceptable solution is

$$
b=0, \quad \theta^{\prime}=3 / 2 .
$$

We also require $Y_{12}^{u} \sim \lambda^{6}$, i.e. $\mathcal{Y}_{12}^{u} \sim \chi^{a} \chi^{\prime(3-a)}$ for $a=0,1,2,3$, and $Y_{12}^{d} \sim \lambda^{6}$, i.e. $\tilde{\mathcal{Y}}_{12}^{d} \sim$ $\chi^{c} \chi^{\prime(3-c)}$ for $c=0,1,2,3$. This leads to the CC equations

$$
\begin{aligned}
& a+(3 / 2)(3-a)-3 \alpha_{2}-3=0, \\
& c+(3 / 2)(3-c)-3 \alpha_{2}-4=0,
\end{aligned}
$$

which immediately implies $a=c+2$. Since $a$ cannot be bigger than 3 we need only consider the cases $c=0$ or $c=1$. Thus we have the solutions

$$
b=0, c=0, a=2, \alpha_{2}=1 / 6, \alpha_{1}=-4 / 3, \theta^{\prime}=3 / 2,
$$

and

$$
b=0, c=1, a=3, \alpha_{2}=0, \alpha_{1}=-1, \theta^{\prime}=3 / 2 .
$$

The last case allows $\mathcal{Y}_{12}^{u} \sim \chi^{\prime 2}$, i.e. $Y_{12}^{u} \sim \lambda^{4}$ which is too big. Therefore the only viable solution corresponds to the first set, eq. (34). For this solution, we find

$$
Y^{u} \sim\left(\begin{array}{ccc}
\lambda^{8} & \lambda^{6} & \lambda^{4} \\
\lambda^{6} & \lambda^{4} & \lambda^{2} \\
\lambda^{4} & \lambda^{2} & 1
\end{array}\right), \quad Y^{d} \sim \lambda^{2}\left(\begin{array}{ccc}
\lambda^{6} & \lambda^{4} & \lambda^{4} \\
\lambda^{4} & \lambda^{2} & \lambda^{2} \\
\lambda^{4} & \lambda^{2} & 1
\end{array}\right)
$$

where, for example, the coefficients of $Y_{33}^{u}, Y_{23}^{d}, Y_{32}^{d}$ and $Y_{22}^{d}$ terms could be small enough to satisfy experimental bounds, and so forth for other entries.

Our explanation for property (iii) also requires that the entire contribution to the lepton matrices arises from higher-dimension operators. A charge assignment which satisfies this requirement is

$$
\begin{gathered}
\bar{q}_{L}+\bar{q}_{e^{c}}+q_{H_{1}}=2\left(a_{1}+a_{2}\right)-1, \\
a_{1}=\alpha_{1}=-4 / 3, \quad a_{2}=\alpha_{2}=1 / 6 .
\end{gathered}
$$

These equations imply that the charge assignments for the lepton sector are as for the downquark sector, i.e. $Y^{e} \sim Y^{d}$ at $M_{X}$. This allows $m_{\tau} \approx m_{b}$ and $m_{\tau} m_{\mu} m_{e} \approx m_{d} m_{s} m_{b}$ at $M_{X}$ and, given the above-mentioned freedom in the coefficients of the powers of $\lambda$, this can produce a viable model for lepton masses.]

Given the charge assignments (38), the three linear equations (24), (25) and (37) can be used to further restrict the solutions to the anomaly constraints (23). For example, for the first 3-parameter family of solutions we require $x=-(1 / 6)(3 z+7)$ and $y=(1 / 6)(9 z-13)$.

\footnotetext{
${ }^{2}$ Since a factor of 3 falls within the accepted range $\lambda<a_{i j}<\lambda^{-1}$, the model of Ref. [23] is an illustration of how one can fit experiment with $Y^{e} \sim Y^{d}$.
} 
To pursue this line of research further, the next step is to investigate how the $\mathrm{U}(1)_{A}$ charge assignments that we have made can be derived from a deeper theory (presumably the underlying string theory). Another topic for study, but one with much weaker constraints, is that of neutrino masses and mixing. Further details will be given in Ref. [20]. This research was partially supported by the NSF Grant PHY-93-09888.

\section{References}

[1] S. Weinberg, in A Festschrift for I. I. Rabi (New York Academy of Sciences, 1977).

[2] H. Fritzsch, Phys. Lett. B73 (1978) 317; Nucl. Phys. B155 (1979) 182.

[3] L. Ibañez and G. G. Ross, Phys. Lett. B332 (1994) 100 (hep-ph/9403338).

[4] L. Ibañez and G. G. Ross, Phys. Lett. B110 (1982) 215; L. Alvarez-Gaumé, J. Polchinski, and M. Wise, Nucl. Phys. B221 (1983) 495.

[5] S. Weinberg, in the Proceedings of the XXVI International Conference on High Energy Physics, 1992 (A.I.P., 1993), 346.

[6] J. Ellis and M. K. Gaillard, Phys. Lett. B88 (1979) 315.

[7] V. Jain, "Gauged Flavor U(1) Symmetries and Models of Fermion Mass Matrices", in the Proceedings of the Workshop on the Origin of Fermion Masses and Mixings, Fermilab, Oct. 1994.

[8] P. Ramond, "Textures and Anomalies" (work with P. Binétruy), in the Proceedings of the Workshop on the Origin of Fermion Masses and Mixings, Fermilab, Oct. 1994.

[9] S. Hawking, Phys. Lett. B195 (1987) 337; Phys. Rev. D37 (1988) 904; S. Giddings and A. Strominger, Nucl. Phys. B306 (1988) 890; S. Coleman, Nucl. Phys. B307 (1988) 867.

[10] M. Green and J. Schwarz, Phys. Lett. B149 (1984) 117.

[11] M. Dine, N. Seiberg and E. Witten, Nucl. Phys. B289 (1987) 589.

[12] V. Jain, ITP-SB-94-38 (hep-ph/9407382).

[13] L. Ibañez, Phys. Lett. B303 (1993) 55 (hep-ph/9205234).

[14] E. Cremmer, B. Julia, J. Scherk, S. Ferrara, L. Giradello, and P. Van Niewenhuizen, Nucl. Phys. B147 (1979) 105; E. Cremmer, S. Ferrara, L. Girardello, and A. van Proeyen, Nucl. Phys. B212 (1983) 413.

[15] See, e.g., J.D. Breit, B.A. Ovrut, and G. Segré, Phys. Lett. 162B (1985) 303; M. K. Gaillard and V. Jain, Phys. Rev. D46 (1992) 1786; Phys. Rev. D49 (1994) 1951; M. K. Gaillard, V. Jain, and K. Saririan, Berkeley-Stony Brook preprint (1994). 
[16] P. Ginsparg, Phys. Lett. B197 (1987) 139.

[17] L. Dixon and V. Kaplunovsky, Nucl. Phys. B355 (1991) 649.

[18] I. Antoniadis, K. Narain, and T. Taylor, Phys. Lett. B267 (1991) 37.

[19] L. Ibańez and D. Lüst, Nucl. Phys. B382 (1992) 305.

[20] V. Jain and R. Shrock, Stony Brook preprint ITP-SB-94-56.

[21] P. Ramond, R. Roberts, and G. G. Ross, Nucl. Phys. B406 (1993) 19 (hep-ph/9303320).

[22] A. Kusenko and R. Shrock, Phys. Rev. D50 (Rapid Commun.) (1994) R30 (hepph/9310307); ITP-SB-93-62 (hep-ph/9401274).

[23] A. Kusenko and R. Shrock, Phys. Rev. D49 (1994) 4962 (hep-ph/9307344). 